\title{
A Comparative study of the Image Registration Process on the Multimodal Medical Images
}

\author{
Nitin Arora1), Mamta Martolia2), Alaknanda Ashok ${ }^{3)}$
}

\begin{abstract}
Registration of image is the process of overlaying one or more image to a reference image of the same image taken at different time, from different view point or taken from different sensor device. The difference in between images is limelight due to different imaging condition which contains yielding in maximum similarity between the input and the reference images. In this paper, we perform a comparative study of the image registration process on the multimodal medical images by using different genetic algorithm. We use simple genetic algorithm, sharing genetic algorithm and GA combined with hill climbing algorithm using two selection criteria as roulette-wheel selection and tournament selection. Results shows that hill climbing algorithm is a local search algorithm and its execution is fast.
\end{abstract}

Keywords: image, registration, genetic algorithm, hill climbing, multimodal, medical.

\section{Introduction}

Registration of image is the process of overlaying one or more image to a reference image of the same image taken at different time, from different view point or taken from different sensor device. The Difference in between images is limelight due to different imaging condition which contains yielding in maximum similarity between the input and the reference images. Image registration geometrically aligns two images, the reference image and captured image. Image registration is a mandatory step in all image analysis tasks in which the final information is gained from the combination of various data sources like in image fusion, change detection, and multichannel image restoration[1]. Mainly registration is required in remote sensing (weather forecasting/monitoring, change detection, image mosaicing, creating

Received(October 13, 2016), Review Result(1st: November 1, 2016, 2nd: December 2, 2016), Accepted(March 10, 2017)

${ }^{1}$ Department of Computer Science and Engineering, KL University

email: nitinarora47@gmail.com

${ }^{2}$ (Corresponding Author) Department of Computer Science and Engineering, KL University email: meet3.16@gmail.com

${ }^{3}$ Department of Computer Science and Engineering, KL University

email: ashok@gmail.com 
super-resolution images, geographic information systems (GIS)), in medicine (combining computer tomography (CT) and NMR data to obtain more complete information about the patient, monitoring tumor growth, treatment verification, in cartography (map updating), and in computer vision (target localization, automatic quality control), to name a few[2-7].

The process of registration involves finding a single transformation imposed on the input image by which it can align with the reference image[8]. It can be viewed as different combination of choice for the following four components: Feature space, Search space, Similarity measure, and Search strategy

The Feature space extracts the information in the images that will be used for matching. The Search space is the class of transformation that is capable of aligning the images. The Similarity measure gives an indication of the similarity between two compared image regions. The Search strategy decides how to choose the next transformation from the search space, to be tested in the search to spatial transformation[9].

This proposed work focuses on registration of images of two medical images of having different modality i.e. image acquired with different sensor e.g. images, MRI images. We consider set of image pixel intensity as the feature space and affine transformation as the search space. A popular similarity measure is mutual information (MI) consider as the mutual information[10]. MI is totally based on the information theory which compares the statistical dependency between images. Registration based on the MI is robust and can be used for a large class of images acquired by the same/different sensors. For the search strategy we use soft computing such as simple genetic algorithm and share genetic algorithm. Genetic algorithm (GA) is based on the concept of the natural process of species evolution to realize simple and robust methods for optimization[11]. To improve the time constraint of the registration process we apply simple genetic algorithm combined with the hill climbing algorithm. Hill climbing algorithm is a local search algorithm and execution is fast[12-13].

The rest of the paper is organized as follows: Section 1 is the Introduction of image Registration. The second section is a literature Survey of the area that gives the reader some theoretical concept about the image registration process. Section 3 reviews the Concepts involved and some of the research work on them concerning about image registration. The fourth section focuses on Implementation issues. The fifth section contains the Results obtained from research. The sixth and last section contains Conclusions and Future work drawn and guidelines on potential future work in the areas.

\section{Concepts}




\subsection{Search Space}

When dealing with the problem of image registration, we work for a best solution which depends on the number of parameters $(\mathrm{n})$ that constitute a solution, an $\mathrm{n}$-dimensional search space consisting of the set of all possible solutions.If we mark each point in the search space with the corresponding cost for that solution, we get a landscape-like hyper surface. The goal is to find the lowest valley in this landscape which often a rather time consuming process, since the hyper surface rarely behaves in a smooth and predictable way. Figure 3.1 shows an interpolated mesh of a landscape generated from a typical image registration problem instance.

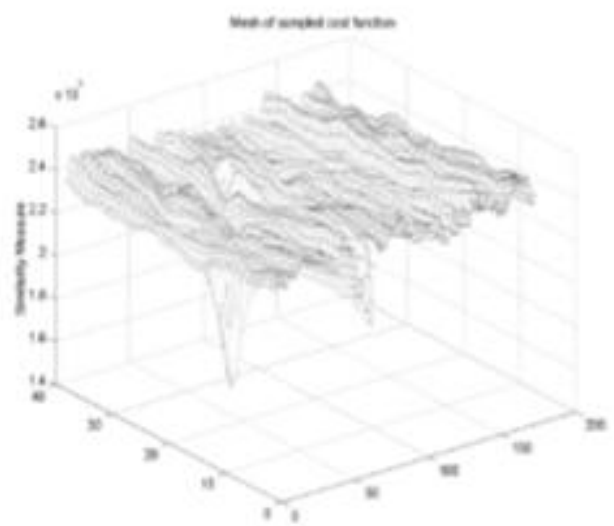

[Fig. 1] Cost function landscape generated from a 2D search space algorithms

\subsection{Genetic Algorithm Description}

The description of genetic algorithm consisting of maintaining a population $x 1 \ldots n=\{x 1 \cdots$ xni\} of $\mathrm{n}$ individuals $\mathrm{xi}$ (which may consist of a vector of parameters). These individuals are candidate solutions to some objective function $\mathrm{F}$ (xi) that is to be optimized to solve the given problem. The individuals are represented in the form of 'chromosomes (pixel)' which are strings defined over some alphabet that encode the properties of the individuals. More formally, using an alphabet $A=\{0,1 \ldots k-1\}$, we define a chromosome $C=\left\{c 1 . \ldots c^{\prime} i\right\}$ of length $\mathrm{l}^{\prime}$ as a member of the set $\mathrm{S}=\mathrm{Al}^{\prime}$, i.e., chromosomes are strings of 1 symbols from $\mathrm{A}$. Each position of the chromosome is called a gene, the value of a gene is called an allele, the chromosomal encoding of a solution is called the genotype, and the encoded properties themselves are called the phenotype of the individual. Being Meta heuristic GA require several 
decision to be made during implementation for encoding, selection, crossover and mutation.

Encoding: The first decision to take when implementing a GA is how solution states should be encoded into chromosomes. Following are the encoding techniques:

Binary Encoding

Octal Encoding

Hexadecimal Encoding

Gray Encoding

Floating Point Encoding

Selection: In selection the offspring producing individuals are chosen. The first step is fitness assignment. Each individual in the selection pool receives a reproduction probability depending on the own objective value and this value of all other individuals is in the selection pool. This fitness is used for the actual selection step afterwards. Some basic terms are used for comparing the different selection schemes. The definition of these terms follows:

Selective pressure: It is the probability selecting the best individual being compared to the average probability of selection of all individuals.

Bias: It is the absolute difference between an individual's normalized fitness and its expected probability of reproduction.

Spread: It is the range of possible values for the number of offspring of an individual.

Selection intensity: It is the expected average fitness value of the population after applying a selection method to the normalized Gaussian distribution.

Selection variance: It is the expected variance of the fitness distribution of the population after applying a selection method to the normalized Gaussian distribution.

\subsection{Stopping Criteria}

Common to most stochastic optimization algorithms, we have no clear way of knowing when to stop the search and accept the currently best solution as the optimal or near-optimal solution. In GA, we usually fix the number of generations to evolve, or end when the algorithm lack to make progress, which is defined e.g. in terms of $G$ number of non-improving generations.

\subsection{A Pseudo-code of Genetic Algorithm}

Procedure GA 
begin

$$
\begin{aligned}
& \text { for } i:=l \text { to } n \text { do begin } \\
& \text { initialize }\left(x_{i}\right) \quad\left({ }^{*}\right. \text { with random values) } \\
& \qquad f\left(x_{i}\right)=\text { evaluate }\left(x_{i}\right) \text { ( }{ }^{*} \text { determine fitness*) } \\
& \text { end }
\end{aligned}
$$

while termination condition not satisfied do begin

$\mathrm{p} 1 \ldots \mathrm{n}:==$ select from $x 1 \ldots \mathrm{n}$ inrandomorder

$$
\begin{gathered}
\text { for } i:=l \text { to } \mathrm{n} \text { step } 2 \text { do begin } \\
x \mathrm{i}^{\prime}, \mathrm{xi}^{+}+1^{\prime}:=\text { crossover }(\mathrm{pi}, \mathrm{pi}+1) \\
\text { mutate }\left(\mathrm{xi}^{\prime}\right) \\
\text { mutate }\left(\mathrm{xi}^{\mathrm{i}}+1^{\prime}\right)
\end{gathered}
$$

end

$$
x_{1 \ldots n}=x_{1 \ldots n}^{\prime} \quad\left(\quad\left({ }^{*} \text { copy new population * }\right)\right.
$$

for $\mathrm{i}:=1$ to $\mathrm{n}$ do begin

$$
\text { f ( xi) :=evaluate }(x i)
$$

end

end $\{\mathrm{f}$ while $\mathrm{g}\}$

end

\subsection{Share Genetic Algorithm}

The Simple-GA is able to explore effectively a multimodal search space. However it tends to find one single optimum, thus it can still be trapped in local optima. One strategy to overcome this problem consists in maintaining population diversity, so that different sub-populations are able to explore different portions of the search space, in order to identify and converge towards different multiple optima. In a multimodal search space, each peak can be thought of as a niche explored by a subpopulation, similarly to nature where there are environments (niches) that can support different types of life (Goldberg, 1989), as depicted in Figure 2.

In nature, a niche is able to support a certain number of individuals depending on the niche fertility and the individual capacity of exploiting this fertility. If there are too many individuals, the niche will not be able to support all of them, and less competitive individuals are likely to die. 


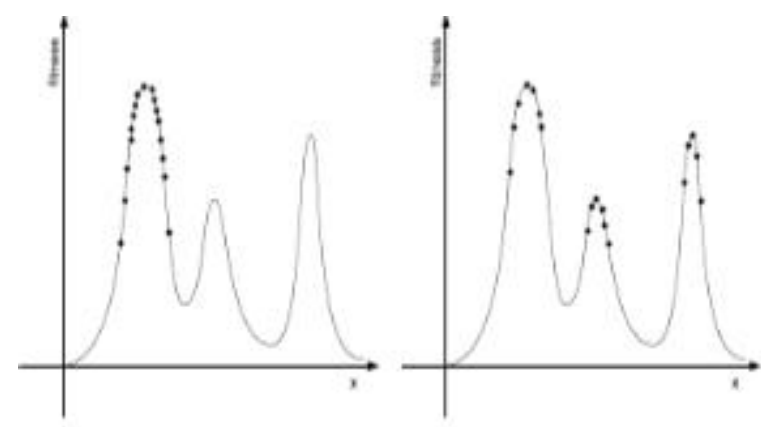

[Fig 2]: The convergence of Simple-GA versus Niche-GA

Differently, if there are too few individuals, they will start to reproduce quickly in order to exploit the niche as measured by the niche peak's fitness relative to the other niche peaks' fitness values present in the domain. Similarly, in genetic algorithms the number of individuals supported by a niche should be proportional to the niche's carrying capacity. In literature several methods have been proposed. One of the mechanism is the fitness sharing (Goldberg and Richardson, 1987). The idea behind the sharing method is to reduce the fitness of individuals that are very similar in their chromosome. In this way, individuals that uniquely exploit portions of the search space are privileged for reproduction, while discouraging redundant individuals in the same area. The method is based on the determination of the shared fitness of the individual $i$ as

$$
f^{\prime}(i)=\frac{f(i)}{m_{i}}
$$

Where $\mathrm{f}(\mathrm{i})$ is the individual's raw fitness and miis the niche count that is defined as

$$
m_{i}=\sum_{j=1}^{n} \operatorname{sh}(d(i, j))
$$

The sharing function sh depends on the distance (dissimilarity) $d$ ( $i$; j) between the individual $\mathrm{i}$ and the individual $\mathrm{j}$. It is a monotonically decreasing function, so that the niche count is reduced if individuals are closer. In particular, it returns 1 if the elements are identical, and 0 if they exceed some threshold of dissimilarity. The function originally proposed by Goldberg (GOLD87) is defined as

$$
\operatorname{sh}(d)= \begin{cases}1-\left(\frac{d}{0_{\text {share }}}\right) & \text { if } d<0_{\text {share }} \\ 0 & \text { otherwise }\end{cases}
$$

Where $\mathrm{d}$ is the distance, and the dissimilarity threshold. 


\subsection{Hill-Climbing Algorithm}

To understand where the name of this technique comes from, imagine that the space of all possible solutions to a given problem is represented as a three-dimensional contour landscape. A given set of coordinates on that landscape represents one particular solution. Those solutions that are better are higher in altitude, forming hills and peaks; those that are worse are lower in altitude, forming valleys. A "hill climber" is then an algorithm that starts out at a given point on the landscape and moves inexorably uphill. Hill-climbing is what is known as a greedy algorithm, meaning it always makes the best choice available at each step in the hope that the overall best result can be achieved this way. It can be used to solve problems that have many solutions, some of which are better than others.

\subsection{A Pseudo code of Hill-Climbing Algorithm}

Hill Climbing Algorithm

$$
\begin{aligned}
& \text { Current Node = start Node; } \\
& \text { Loop do } \\
& \text { L= NEIGHBORS (Current Node); } \\
& \text { nextVal= -INF; } \\
& \text { nextNode=NULL; } \\
& \text { for all } \mathrm{x} \text { in L } \\
& \text { if }(\text { EVAL }(\mathrm{x})>\text { nextEval) } \\
& \text { nextNode }=\mathrm{x} \text {; } \\
& \text { nextEval= EVAL }(\mathrm{x}) \text {; } \\
& \text { if nextEval<= EVAL(CurrentNode) } \\
& \quad \text { return CurrentNode; }
\end{aligned}
$$

CurrentNode= nextNode;

\section{Implementation}

The experiment is to be tested in MATLAB 7.0. The registration process is implemented for the multimodal images. The implementation aspects of these steps are as follows:

\subsection{Selection of Feature space}


A Comparative study of the Image Registration Process on the Multimodal Medical Images

In this step of the image registration we have to find the information from the image by which matching can be done. In this work we use pixel intensity as the feature space.

\subsection{Selection of Search space}

The class of transformation that is assumes to be capable of aligning the input image with the reference image. We use affine transformation as the search space. This transformation is useful when registering images taken from a distant platform of a flat scene. We use translation, rotation and scaling as the transformation parameter.

\subsection{Selection of Similarity Measure}

Multimodal images of the same scene represent measurements of different properties of the objects in that scene. Mutual information measures the degree of statistical dependency of two random variables. It can be used to measure how well one image explain the other. For two data set $\mathrm{A}=\{\mathrm{a}\}$ and $\mathrm{B}=\{\mathrm{b}\}, \mathrm{MI}$ is defined as Mutual information is related to the information theoretic notion of entropy by the following equations

$$
\begin{aligned}
I(A, B) & =H(A)+H(B)-H(A, B) \\
= & H(A)-H(A / B) \\
= & H(B)-H(B / A)
\end{aligned}
$$

with $H(A)$ and $H(B)$ being the entropy of $A$ and $B$ respectively, $H(A, B)$ their joint entropy, and $\mathrm{H}(\mathrm{A} / \mathrm{B})$ and $\mathrm{H}(\mathrm{B} / \mathrm{A})$ the conditional entropy of $\mathrm{A}$ given $\mathrm{B}$ and of $\mathrm{B}$ given $\mathrm{A}$, respectively. $\mathrm{H}(\mathrm{A}), \mathrm{H}(\mathrm{A} / \mathrm{B})$ and $\mathrm{H}(\mathrm{A}, \mathrm{B})$ are defined as:

$$
\begin{aligned}
& H(A)=-\sum a P_{A}(a) \log p_{A}(a) \\
& H(A \mid B)=-\sum a, b P_{A, B}(a, b) \log p_{A \mid B=b}(a) \\
& H(A, B)=-\sum a, b P_{A, B}(a, b) \log p_{A B}(a, b) \\
& \quad I(A, B)=\sum a b P_{A B}(a, b) \log \frac{P_{A B}(a, b)}{P_{A}(a) P_{B}(b)}
\end{aligned}
$$




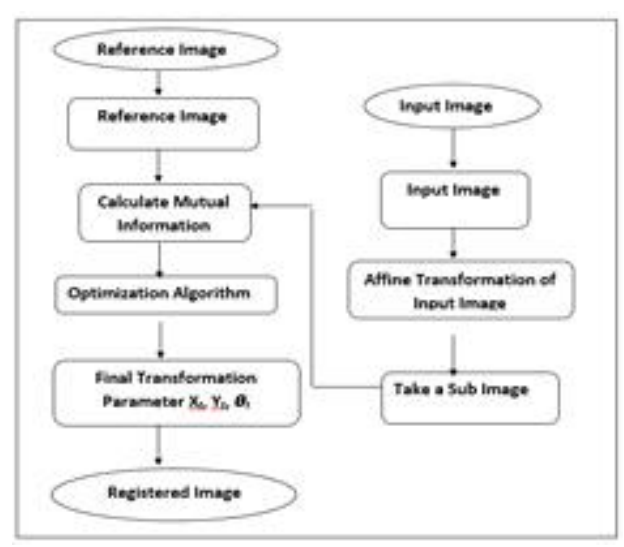

[Fig. 3]Flow Graph of Image Registration using mutual information

\subsection{Algorithmic description}

Assign N A and NB array for bins in the join this to gram, with NA and NB as the number of intensities in the images.

Compute the joint intensity histogram $\mathrm{HIS}(\mathrm{a}, \mathrm{b})$ of the overlap part $\mathrm{A} \cap \mathrm{B}$ in the image by binning the intensity value in $\mathrm{Ais} A(\mathrm{i})=\mathrm{x}$ and the corresponding value in $\mathrm{B}$ is $\mathrm{B}(\mathrm{i})=\mathrm{b}$ then $\operatorname{HIS}(a, b)=\operatorname{HIS}(a, b)+1$.

Calculate the normalized joint histogram

$$
\operatorname{PDF}(a, b)=\frac{H I S(a, b)}{\sum H I S a, b(a, b)}
$$

Calculate the marginal intensity distribution by the sum of the rows and columns of normalized joint histogram

$$
\begin{aligned}
& P_{A}(a)=\sum b P D F(a, b) \\
& P_{B}(b)=\sum a P D F(a . b)
\end{aligned}
$$

Calculate mutual information. 


\subsection{The proposed Algorithm}

Input $=$ Captured image

Output=Registered image

Step 1:- Firstly Image is captured by the high resolution camera.

Step 2:- Then image is divided into well-defined blocks.

Step 3:- Captured image is converted into grayscale image.

Step 4:- Calculate the gradients Gx, Gy of the captured image and Gx', Gy' of the referenced image.

Step 5:- Scaling and Rotation of captured image is done.

Step 6:- Calculate the pixels of both images i.e. Captured image and reference image.

Step 7:- Each position of the chromosome (pixel) is called a gene; the value of a gene is called an "Allele".

Step 8:- Calculate the mutual information (MI) from both images (Alleles and reference image Alleles.)

Step 9:- Then "Allele" based matching is done.

Step 10:- if Captured image $=$ reference image

Then

Captured becomes registered image.

Else

Not registered image.

\section{Results}

We tested 7 pair of medical images for image registration using the following algorithm.

Simple Genetic Algorithm using roulette-wheel selection (GAr).

Shared Genetic algorithm using roulette-wheel selection (SGAr).

Shared Genetic algorithm using tournament selection (SGAt).

To illustrate the performance of our algorithm, we consider two type of medical images CT images and MRI images of the same patient. We take these images from the medical image database MEDIPIX. Table1, Table2, Table3, shows the result of the three algorithm for the three images such as amount of translation along the $\mathrm{x}$-axis and $\mathrm{y}$-axis and rotation angle required 
to achieve the registration. Maximum mutual information (MMI), the error and the time elapsed is shown below. The output image is also shown in the fig below. In the output image Image 1 is the reference image, Image2 is the captured image and registered image is the after registration. The experiment using the termination criteria of 50 generation with arithmetic crossover and mutation rate of .01 .

\begin{tabular}{|c|c|c|c|c|c|c|}
\hline lates & 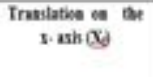 & 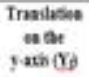 & 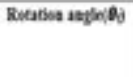 & Marlass= MII & Emor & Tase elapied \\
\hline Deg 5.1 & 515152 & 45 क्स & $10 \times 01$ & 11585 & 52580 & 47673004 \\
\hline nes? & 16.7525 & 34517 & -75314 & 0 S24! & 11314 & 117,645594 \\
\hline Fig 53 & 15.3042 & 82494 & 53525 & 0.432 & 3.0431 & 5921507 \\
\hline
\end{tabular}
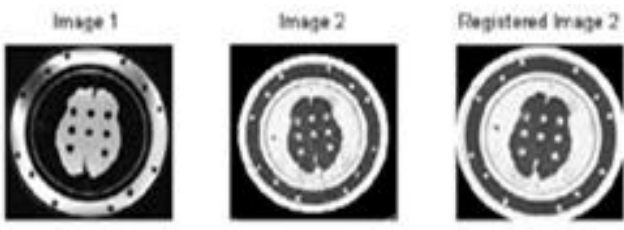

[Fig. 4] Result of Simple Genetic Algorithm using roulette-wheel selection (GAr)
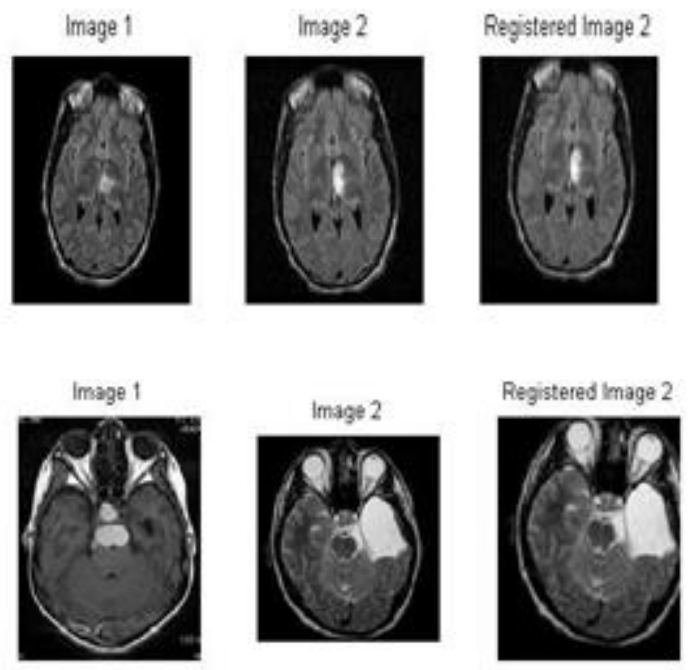
A Comparative study of the Image Registration Process on the Multimodal Medical Images

\begin{tabular}{|c|c|c|c|c|c|c|}
\hline Laten & $\begin{array}{c}\text { Traslativa ot } \\
\text { the } \\
\operatorname{rasis}\left(X_{d}\right)\end{array}$ & $\begin{array}{l}\text { Intslatica } \\
\text { catte } \\
\text { yutivid }\end{array}$ & $\begin{array}{l}\text { Retatioa } \\
\text { apple(a) }\end{array}$ & Mtriem MII & Entrot & Tane duped \\
\hline Fig 5.4 & $\$ 05196$ & 5,4520 & 10283 & 11565 & $56+73$ & 45.72856 \\
\hline Figss & 15.0596 & $764 \%$ & .11.126 & 0.528 & 11349 & $12057 \times 4$ \\
\hline Fiद 56 & 12781 & 56916 & 6765 & 0.555 & $2 m 22$ & 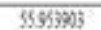 \\
\hline
\end{tabular}
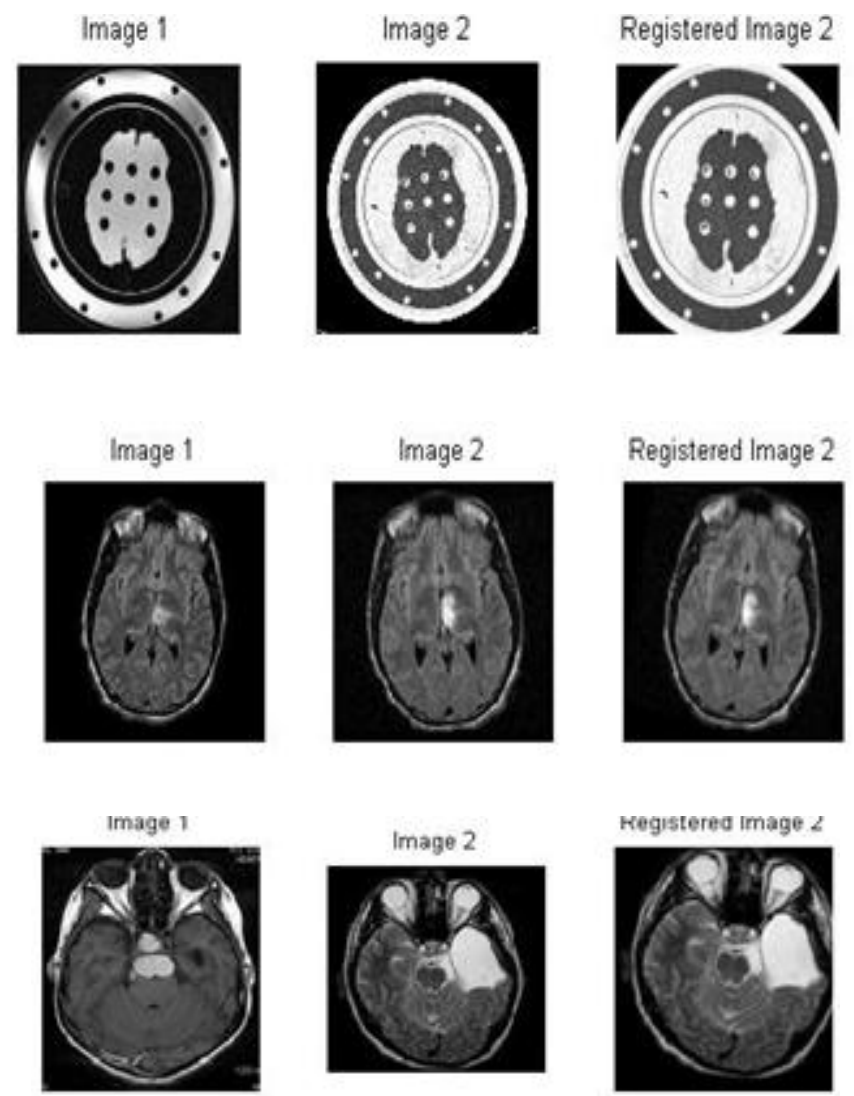

[Fig. 5] Result of shared Genetic Algorithm using roulette-wheel selection (SGAr)

\section{Conclusion and Futurework}

\subsection{Conclusion}

In this paper, we implement two genetic algorithms with two selection criteria i.e. simple genetic algorithm and shared genetic algorithm. We implement a hybrid genetic algorithm 
which is combined with hill climbing algorithm. We conclude from the extracted result as follows:

\begin{tabular}{|c|c|c|c|c|c|c|}
\hline Inder & $\begin{array}{c}\text { Translation on be } \\
\text { I-aris }(\Delta)\end{array}$ & 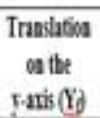 & Rotation angle $\left(\theta_{1}\right)$ & Slarimon YII & Emor & $\begin{array}{c}\text { Time } \\
\text { thapsed }\end{array}$ \\
\hline Fig 5.13 & 31.4870 & 65.5278 & 93194 & 1.1551 & 5358 & 5428630 \\
\hline Fig 5.14 & 146707 & 7.1799 & -10.6536 & 0.8315 & 11355 & 58.18554 \\
\hline Fig 5.15 & 159948 & 8.1671 & .92246 & 0.8414 & 30692 & 58.797584 \\
\hline
\end{tabular}
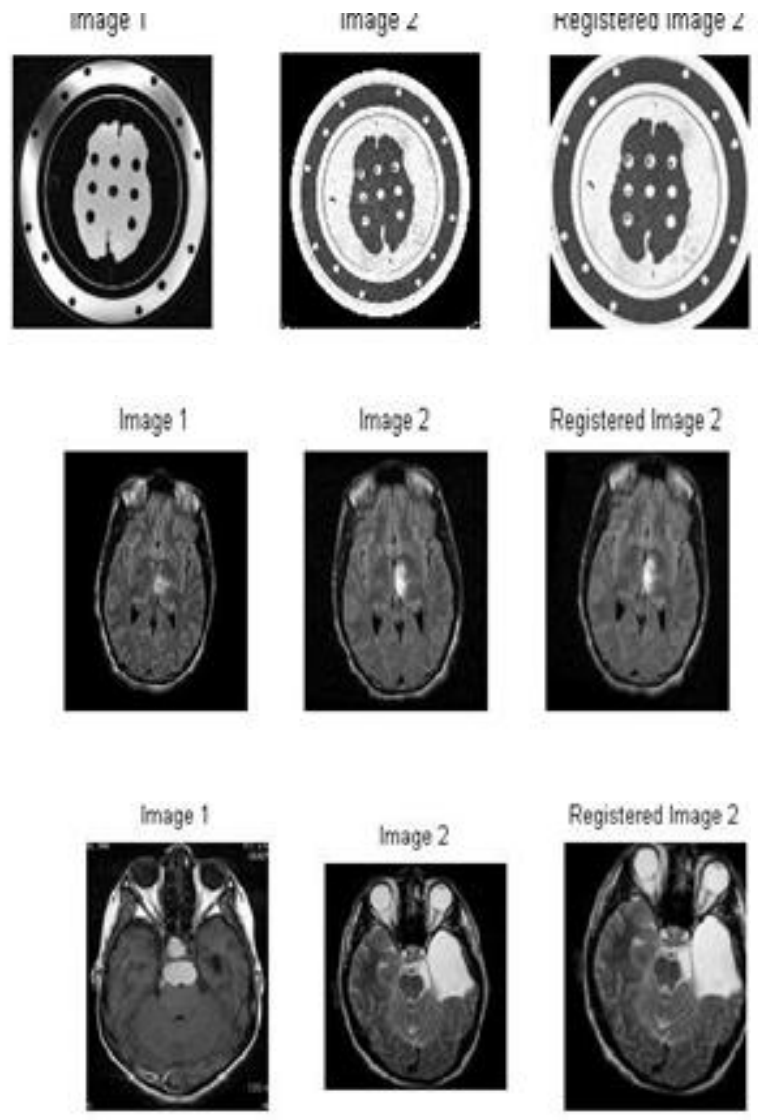

[Fig. 6] Result of shared Genetic Algorithm using tournament selection (SGAt)

All the three algorithms, simple genetic algorithm, sharegenetic algorithm, and genetic algorithm combined with hill climbing algorithm are feasible alternative in performing image registration. 


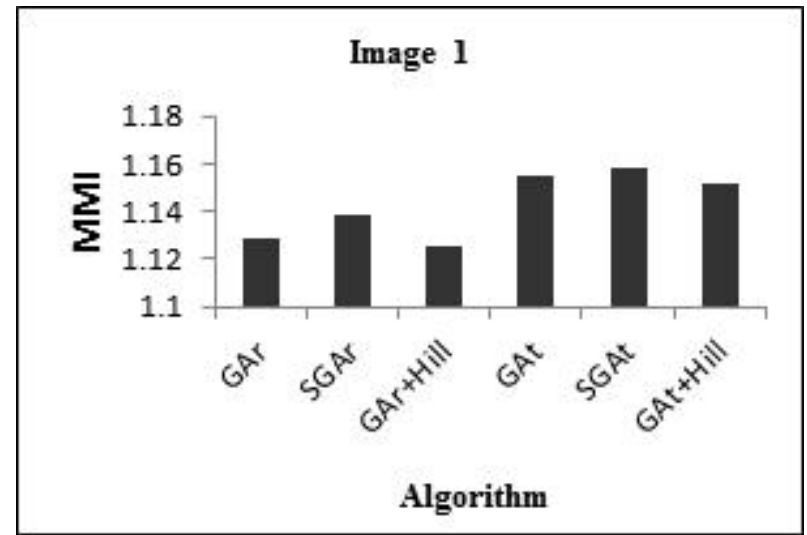

[Fig. 7] MMI value of GAr Algorithm

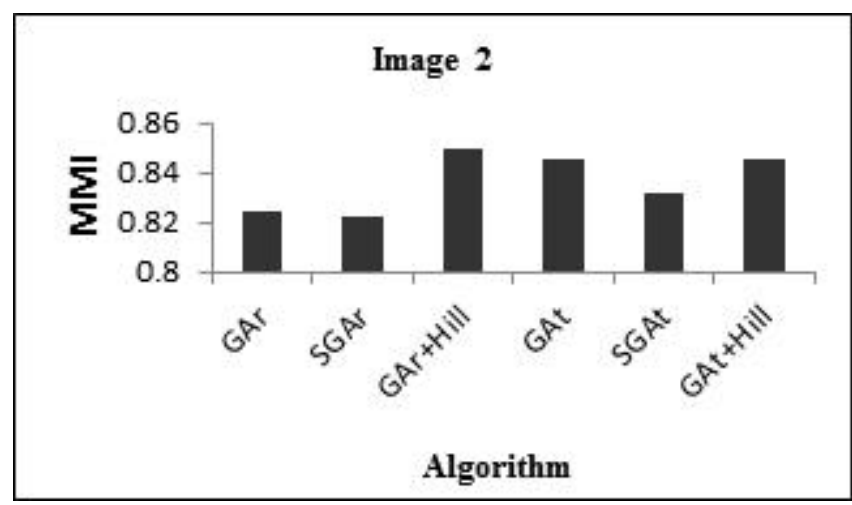

[Fig. 8] MMI value of SGAr Algorithm

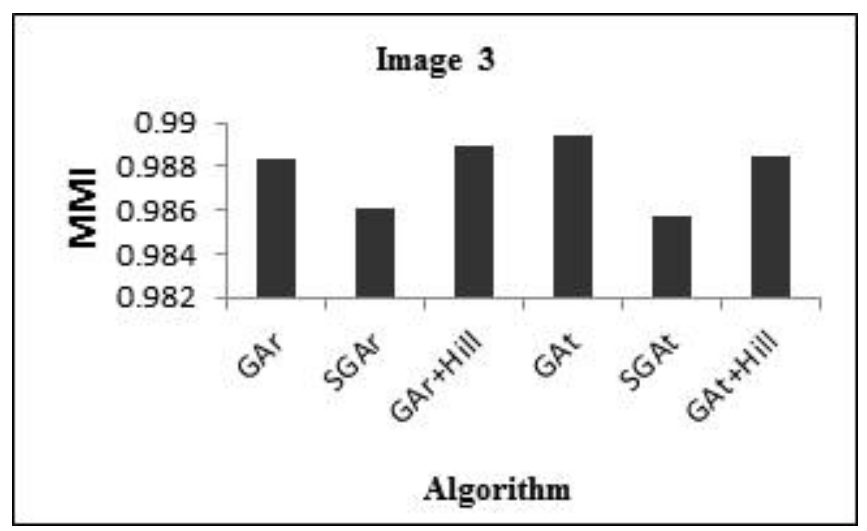

[Fig. 9] MMI value of SGAt Algorithm

Genetic algorithm represents an effective technique in multimodal optimization problem. The 
problem with genetic algorithm is that it can be fascinated in local minimum due to genetic drift which is solved using sharing genetic algorithm. So that sharing genetic algorithm has given better performance than the genetic algorithm, but the algorithm is highly sensitive to calibration parameter. Therefore some time it does not give better performance.

Sequentially we use genetic algorithm and hill climbing algorithm. First the genetic algorithm executes after that the hill climbing algorithm where genetic algorithm gives near to option. As hill climbing algorithm is local search algorithm this may occur that the maxima which is achieved in genetic algorithm has no improvement after the hill climbing algorithm.

\subsection{Future Work}

In future work one can use mutual information as the similarity measure, other similarity measure such as gradient coded MI; weighted MI can also be used. We can also use Parallel GA to improve performance and we can also use chromosome vector of four parameter translation along the $\mathrm{x}$-axis and $\mathrm{y}$-axis, rotation, scaling.

\section{References}

[1] B. Zitová, J. Flusser, Image Registration Methods: A Survey, Image and Vision Computing, (2003), Vol.21 pp.977-1000.

[2] M. P. Wachowiak, R. Smolíková, Y. Zheng, J. M. Zurada, and A. S. Elmaghraby. An Approach to Multimodal Biomedical Image Registration Utilizing Particle Swarm Optimization, IEEE Transactions On Evolutionary Computation, (2004), Vol.8, No.3, pp.289-301.

[3] Jamil Ahmad, Muhammad Sajjad, Irfan Mehmood, Seungmin Rho, Sung Wook Baik, "Describing Colors, Textures and Shapes for Content Based Image Retrieval - A Survey," JOURNAL OF PLATFORM TECHNOLOGY VOL. 2, NO. 4, DECEMBER 2014, pp.34-48

[4] Woo-Chul jung, Sueng-Soo Lee, Joon-Hoon Park, Design of optimal snowmelting system with snowfall image processing based on the IoT technology, Asia-pacific Journal of Multimedia Services Convergent with Art, Humanities, and Sociology, ISSN:2383-5281, Vol.5, No.6, December (2015), pp.521-530, http://dx.doi.org/10.14257/AJMAHS.2015.12.38

[5] Ji-Hun Lee, Doo-Hyun Choi, Palm Lines Extraction on a High Resolution Image using Mosaic-like Method, Asia-pacific Journal of Multimedia Services Convergent with Art, Humanities, and Sociology,ISSN:2383-5281, Vol.6, No.3, March (2016), pp. 9-17, http://dx.doi.org/10.14257/AJMAHS.2016.03.07

[6] Ji-Hun Ha, Hyo-Hyuk Im, Yong-Hyuk Kim, Counting Harmful Aquatic Organisms in Ballast Water through Image Processing, Asia-pacific Journal of Multimedia Services Convergent with Art, Humanities, and Sociology, ISSN:2383-5281, Vol.6, No.3, March (2016), pp. 383-391, http://dx.doi.org/10.14257/AJMAHS.2016.03.13 
[7] Ho-Sun Shin, Kang-Hee Lee, A proposal of visualization method of MBTI personality types using celebrity images, Asia-pacific Journal of Multimedia Services Convergent with Art, Humanities, and Sociology, ISSN:2383-5281,Vol.6, No.8, August (2016), pp. 491-498, http://dx.doi.org/10.14257/AJMAHS.2016.08.32

[8] G. Egnal and K. Daniilidis, Image Registration Using Mutual Information, University of Pennsylvania Department of Computer and Information Science Technical, Report No. MS-CIS-00-05, (2005).

[9] F. Maes, D. Vandermeulen, and P. Suetens, Medical Image Registration Using Mutual Information, Proceedings Of The Ieee, (2003) October, Vol.91, No.10, pp.1699-1722.

[10] W. R. Crum, T. Hartkens, and D. L. G. Hill, Non-rigid image registration: theory and practice, The British Journal of Radiology, (2004), Vol.77, pp.S140-S153.

[11] A. Sotiras, C. Davatzikos, and N. Pragios, Deformable Medical Image Registration: A Survey, IEEE Transactions on Medical Imaging, (2013), Vol.32, No.7, pp.1153-1190.

[12] S. Baker, D. Scharstein, J. P. Lewis, S. Roth, M. J. Black, and R. Szeliski, A database and evaluation methodology for optical flow, Int. J. Comput. Vis., (2010), Vol.92, No.1, pp.1-31.

[13] M. Holden, A review of geometric transformations for nonrigid body registration, IEEE Trans. Med. Imag., (2008), Vol.27, No.1, pp.111-128. 